Aus der entwicklungsgeschichtlichen Abteilung des anatomischen Instituts zu Breslau.

\title{
Die Entwicklung der Nasenhöhle bei Amphibien.
}

\author{
Teil III: Gymnophionen. \\ Von \\ Privatdozent Dr. V. Hinsberg,
}

Assistent an der Universitäts-Poliklinik für Ohren-, Nasen- und Kehlkopf-

krankheiten zu Breslau.

Hierzu Tafel XIX.

Bei der Seltenheit der Gymnophionen war es mir leider nicht möglich, ein so einheitliches und lückenloses Material zu erhalten, wie es mir bei Anuren und Urodelen zur Verfügung stand. Für die Untersuchung der ersten Anlage der Nasenhöhle benutzte ich Embryonen von Hypogeophis rostratus, während ich spätere Entwicklungsstadien an Embryonen und jungen Larven von Ichthyophis glutinosus studieren konnte. Die ersteren verdanke ich, wie schon erwahnt, der Liebenswürdigkeit des Herrn Prof. Brauer; es sei mir gestattet, ihm auch an dieser Stelle meinen herzlichsten Dank für die Ueberlassung des so wertvollen Materials auszusprechen.

Das ausgezeichnet conservierte Material von Ichthyophis, aus der Sarasin'schen Sammlung stammend, stellte mir Herr Dr. Peter in Form von Serien freundlichst zur Verfügung.

Meine Untersuchungen erstrecken sich demnach auf zwei Gattungen, deren Entwicklung trotz ihrer nahen Verwandtschaft in einigen Punkten von einander abweicht. (cf. Brauer p. 505). Ich glaube jedoch nicht, dass sich diese Differenzen auf so wichtige Anlagen, wie die der Sinnesorgane, erstrecken, thatsächlich zeigt auch bezüglich der Ausbildung des Geruchsorganes der jüngste der von mir untersuchten Embryonen von Ichthoyphis 
eine grosse Uebereinstimmung mit dem altesten Stadium von Hypogeophis.

Wesentliche Fehler dürften deshalb durch diese Ungleichheit des Materials wohl kaum bedingt sein. Aeltere Larven von Gymnophionen habe ich nur bezüglich weniger Punkte genau untersucht, da gerade diese Stadien durch die Arbeiten von P. und F. Sarasin so genau bekannt sind, dass weitere Studien über dieselben wohl kaum mehr nötig sind.

Ich beschreibe, wie bei Anuren und Urodelen, zunächst die erste Anlage des Geruchsorgans, dann die Bildung des Lumens und der Choanen, und endlich die weitere Entwicklung und Blindsackbildung.

\section{A. Erste Anlage der Geruchsplatte.}

\section{a. Stadienbeschreibung.}

\section{Stadium 1 .}

(Hierzu Figur 1.)

Embryo von Hypogeophis rostratus, in der ausseren Entwicklung dem in Figur 13 von Brauer (7) abgebildeten Stadium entsprechend. Horizontalschnittserie zu $8 \mu$.

An einzelnen Stellen ist am Ektoderm deutlich Deckschicht und Sinnesschicht zu unterscheiden, stellenweise hat erstere bei der Conservierung anscheinend etwas gelitten, wenigstens ist sie nicht überall als solche zu erkennen. Die Sinnesschicht bildet ventral vom primären Augenbläschen eine flache Verdickung, die Geruchsplatte. Während die innere Zellage des Ektoderms an den übrigen Stellen aus einer Schicht cubischer Zellen besteht, wird die Geruchsplatte aus zwei bis drei Reihen cubischer bis cylindrischer Zellen gebildet. Die Deckschicht ist am Aufbau der Geruchsplatte $\mathrm{nich}$ t beteiligt. Sie zieht über die Ränder derselben, deutlich gegen die Sinneszellen abgrenzbar, hinweg. Ueber dem Centrum der Sinnesplatte jst sie nicht an allen Schnitten erkennbar, jedoch finden sich in einzelnen hier schlecht gefärbte Zellen, die wir wohl, ähnlich wie bei den Urodelen, als zu Grunde gehend zu deuten haben. (Fig. 1 bei $\mathrm{x}$ ).

Die Geruchsplatte ist mit ihrer medialen Fläche dem Gehirn dicht benachbart, sie ist vollkommen flach, von einem Grübchen ist noch nichts wahrnehmbar. 


\section{Stadium 2.}

Embryo von Hypogeophis rostratus, ungefăhr der Figur 15 von $\mathrm{Brauer}$ entsprechend. Horizontalschnittserie zu $8 \mu$.

Die Deckschicht verhălt sich wie beim eben beschriebenen Stadium. Die Geruchsplatte hat an Umfang etwas zugenommen, ihr apicaler Pol ist etwas nach innen eingebuchtet.

\section{Stadium 3.}

(Hierzu Figur 3).

Embryo von Hypogeophis rostratus, in der äusseren Entwicklung der Fig. 23 von Brauer entsprechend. Lückenhafte Horizontalschnittserie zu $8 \mu$.

Ueber der Geruchsplatte hat sich ein lăngliches Grübchen gebildet, und zwar anscheinend dadurch, dass die Ränder !der Platte mit dem Ektoderm der Umgebung, das durch die beginnende Mesodermwucherung peripherwarts ausgedehnt wurde, nach aussen gerückt sind, während die mittlere Partie dem Gehirn dicht benachbart blieb. Sie stellt also jetzt ein nach aussen concaves Gebilde dar.

Das so entstandene Grübchen ist am dorsalen Pol am tiefsten, während es sich oralwärts allmăhlich abflacht. Die Deckschicht zieht bis zum Rande des Grübchens und hört hier. auf, im Bereich des Grübchens fehlt sie, so dass die Sinneszellen unmittelbar an der Obertläche liegen. (Fig. 2).

\section{b. Zusammenfassung der Ergebnisse, und Vergleich der} Befunde bei Gymnophionen und bei Anuren and Urodelen.

Bei den Gymnophionen erfolgt, wie bei den Anuren und Urodelen, die erste Anlage der Geruchsplatte durch eine Verdickung des Ektoderms, bei der ausschliesslich die Sinnesschicht desselben beteiligt ist. Auch bei den Blindwühlen lässt sich mit aller Sicherheit feststellen, dass eine aktive Beteiligung der Deckschicht durch Wucherung ihrer Zellen oder durch Verwachsung mit der Sinnesschicht nicht vorhanden ist.

Im Stadium 1. ist die Deckschicht über dem Rande der Geruchsplatte deutlich erkennbar, über dem Centrum derselben befinden sich schlecht gefärbte Zellen, die wir wohl als den Rest des Teloderms zu deuten haben. In den späteren Stadien zieht sie 
bis zum Rande des Geruchsgrübchens, um hier aufzuhören, wahrend im Bereich des Grübchens selbst die Sinnesplatte unmittelbar an der Oberfläche liegt.

Sinnes- und Deckschicht verhalten sich also bei den Gymnophionen genau so, wie bei den übrigen von mir untersuchten Amphibien.

Bezüglich der Form der Geruchsplatte ist ein nicht unwesentlicher Unterschied zwischen den Blindwühlen und den Anuren und Urodelen zu konstatieren. Während bei den letzteren die Anlage des Geruchorgans sehr bald eine knopfförmige Gestalt annimmt, (cf. Teil 1, p. 433), bleibt sie bei den Gymnophionen ziemlich flach und relativ dünn.

Auch die Bildung des Grübchens erfolgt in verschiedener Weise; bei den Schwanzlurchen und Batrachiern entsteht dasselbe als Aushöhlung an der Oberfläche der Geruchsplatte, wăhrend bei den Gymnophionen die Geruchsplatte als Ganzes sich umbiegt, so dass über ihrer Mitte eine Grube entsteht.

In der Literatur habe ich mikroskopische Untersuchungen über die erste Anlage des Geruchsorgans bei Gymnophionen nicht aufgefunden.

\section{B. Bildung des Nasenlumens and Durchbruch in die Mundhöhle.}

\section{a. Literatar.}

Die erste Anlage der Nasenhöhle und die Lumenbildung, soweit sich dieselbe durch Betrachtung des Oberflächenbildes feststellen lässt, ist bereits von $\mathrm{Brau}$ er in eingehender Weise geschildert worden in Zusammenhang mit der Entstehung des Mundhöhlendaches.

Da das Verständnis der zum Teil sehr complizierten mikroskopischen Bilder wesentlich durch die Kenntnis der ausseren Form erleichtert wird, halte ich es für zweckmässig, zunächst die Resultate der Brauer'schen Untersuchungen kurz zusammenzustellen, um mich bei der Beschreibung meiner Befunde auf dieselben beziehen zu können. Weiter unten werde ich dann zu prüfen haben, wie weit unsere beiderseitigen Befunde sich decken.

Nach Brauer entstehen die ersten Anlagen der Riechgrübchen „als sehr schwache Abflachungen von etwa dreieckiger 
Form", zunächst kaum erkennbar. Dieselben liegen apicalwärts von einer halbkugeligen Vorwölbung, welche der Augenblase entspricht. Etwas später werden sie tiefer und erhalten eine deutliche Umrandung. Sie stehen nicht der Langsachse des Kopfes parallel, vielmebr etwas schief, ihre Lăngsachsen convergieren ventralwärts. Die Grübchen nehmen sodann an Grösse und Tiefe zu, ihre tiefste Stelle „liegt der dorsalen Seite năher als der ventralen", ventralwarts flachen sie sich allmăhlig $a b$, sind aber auch hier scharf umrandet, "besonders von der Mundbucht völlig abgeschlossen." "Der laterale und der mediale Stirnfortsatz, wie man die die Nasenanlage begrenzenden Teile der Stirnflåche hezejchnet, sind mithin ventral vereinigt." Zwischen beiden bildet sich allmăhlig eine Rinne, welche die Riechgrube in direkte Kommunikation mit der Mundbucht bringt, die Nasenrachenrinne. " Die beiden Stirnfortsătze werden so von einander getrennt, der laterale verwăchst mit dem Oberkieferfortsatz, der mediale wuchert in die Tiefe und bildet die Anlage des Gaumens. „Nach der Ablösung der Gaumenanlage wächst der mediale wieder dem mit dem Oberkieferfortsatz vereinigten lateralen entgegen (Fig. 36 a) und legt sich ihm eng an. Dadurch wird natürlich die Nasenrachenrinne verengt bis zu einem allerdings tiefen Spalt. Indem dann die genannten Fortsătze mit einander verschmelzen, verschwindet die aussere Rinne vollstăndig, die Oeffnungen der Riechgruben kommunizieren allein durch einen engen Kanal mit der Mundbucht". Die ăusseren Nasenöffnungen verengen sich im Lauf der Entwicklung.

Diese Befunde Brauers habe ich an den gleich zu beschreibenden Stadien mikroskopisch nachgeprüft. Ich werde mich bei der Schilderung der Befunde der von Brauer gewählten Bezeichnungen "lateraler- und medialer Stirnfortsatz" bedienen, um nicht durch andere Benennungen den Vergleich unserer Resultate zu erschweren. Ich möchte dabei jedoch mit Hinweis auf die Untersuchungen von Peter (22) bemerken. dass die genannten Gebilde nicht genau den Stirnfortsătzen der Amnioten entsprechen. Während nämlich bei den letzteren die Stirnfortsătze die Geruchsgrube umfassen, liegt diese bei den Gymnophionen ganz innerhalb des medialen Stirnfortsatzes. 


\section{b. Eigene Beobachtungen.}

1. Stadienbeschreibung.

\section{Stadium 4.}

Embryo von Hypogeophis rostratus, dem von Brauer in Fig. 32 abgebildeten Entwicklungsstadium entsprechend. Horizontalschnittserie zu $8 \mu$.

Die Geruchsgrübchen hahen an Umfang und Tiefe zugenommen. Am dorsalen $\mathrm{Pol}$ ist ein kleines Lumen entstanden, während das Grübchen mundwärts ganz allmäblig flacher wird. Die Verwachsung der beiden Stirnfortsătze in der im nächsten Stadium näher zu beschreibenden Weise hat begonnen.

\section{Stadium 5.}

(Hierzu Figur 3-5).

Embryo von Hypogeophis, der ausseren Fntwicklung nach dem von $\mathrm{Brauer}$ in Fig. 3ó abgebildeten entsprechend. Horizontalschnittserie zu $8 \mu$.

Der linke Geruchsack und seine Umgebung wurden bei 150facher Vergrösserung modelliert. Fig. 3 stellt die Aussenfläche des Modells dar, und zwar von aussen und unten gesehen, sodass ein Teil der lateralen Kopfflache und des Gaumendaches zur Ansicht gebracht ist. Bei Betrachtung des Modells von dieser Seite făllt zunăchst die Geruchsgrube (gr.) ins Auge. Dieselbe bildet an ihrem dorsalen Ende einen kleinen Blindsack, während sie sich mundwärts allmăhlig abflacht. Der "laterale Stirnfortsatz" ist als Wulst an der nuchalen Umrandung des oralen Teils der Geruchsgrube deutlich zu erkennen. Gegen den medialen ist er durch eine Rinne (Fig. 3 R) scharf abgesetzt. Diese lässt sich oralwärts bis aufs Gaumendach verfolgen und erweitert sich hier zu einem Grübchen (Gr.), das von der Basis der beiden Stirnfortsătze umschlossen wird.

Betrachten wir das Modell von innen her (Fig. 4) - das Gehirn wurde, um einen freien Einblick zu gewinnen, entfernt, so sehen wir zunăchst, apicalwärts vom Auge (Au.), die Geruchsplatte (gs.) als mächtiges, knopfförmiges Gebilde nach innen hervorragen. Unter dem Auge zieht eine Epithelfalte (F.) oralwärts, um in das Epithel des Gaumendaches überzugehen. An der Stelle, an der beide zusammenhängen, zeigt das Gaumendach eine leichte Einbuchtung nach innen $z u$, die, wie ein Ver- 
gleich mit der Aussenfläche des Modells ergiebt, dem vorher erwähnten Grübchen (Fig. $3 \mathrm{Gr}$.) entspricht. Während also die Epithelfalte oralwärts ins Mundhöhlendach übergeht, hängt sie lateralwärts einerseits mit dem Ektoderm des ausseren Stirnfortsatzes, andererseits mit dem des medialen zusammen.

Die Durchsicht der Schnittserie ergiebt einige Details, die für die Deutung dieser Befunde wichtig sind. In Fig. $\tilde{\mathbf{b}}$ ist ein Schnitt wiedergegeben, der durch den kaudalen Pol der Geruchsplatte gelegt ist. $R$ entspricht der Rinne an der Aussenfläche des Modells (Fig. $3 \mathrm{R}$ ), $\mathrm{F}$ der von derselben nach innen ziehenden Falte (Fig. 4 F). Die letztere besteht aus einer doppelten Epithelschicht, die continuirlich mit der Sinnesschicht (s) zusammenhängt. Die Deckschicht (d) des lateralen Stirnfortsatzes (I St) lässt sich bis $R$ deutlich verfolgen, um hier aufzuhören.

Die Falte (F) bildet entschieden die wesentlichste Veranderung gegenüber dem zuletzt beschriebenen Stadium. Bei der Frage, wie die Entstehung derselben zu erklären sei, sind zwei Möglichkeiten in Betracht zu ziehen. Einmal wäre es denkbar, dass die Sinnesschicht in einem der Rinne (R) entsprechenden Bezirk in die Tiefe gewuchert sei, eine Leiste bildend, an deren Entwicklung die Deckschicht ganz unbeteiligt ist. Dabei bliebe freilich unerklärt, wodurch dieses Epithelwachstum bedingt ist.

Als zweite Entstehungsart kommen Wachstumsverschiebungen zwischen lateralem und medialem Stirnfortsatz in Betracht. Besonders bei Besichtigung der Aussenfläche des Modells gewinnt man den Eindruck, als sei der erstere spitzenwärts gewachsen, sich dabei dem medialen in einem kleinen Bezirk anlegend, und als sei die Sinnesschicht beider in dieser Ausdehnung verschmolzen, indem die Deckschicht beider Fortsätze zu Grunde ging. Die Rinne (R) und das Grübchen (Gr.) würden dann die Grenze zwischen beiden Fortsätzen noch markieren.

Welche von diesen Entstehungsarten die richtige ist, oder ob vielleicht eine Kombination von beiden anzunehmen ist, wage ich nicht mit Bestimmtheit zu entscheiden, ich halte jedoch die zuletztbeschriebene für wahrscheinlicher. Dafür spricht, wie gesagt, die Betrachtung des Aussenreliefs des Modells, daneben aber auch ein Vergleich mit dem nächsten Stadium. Derselbe ergiebt, dass ein Wachstum des lateralen Stirnfortsatzes spitzen- 
warts und eine Verschmelzung mit dem medialen thatsächlich stattfindet, es ist also wohl naheliegend, den erwähnten Befund als den Beginn dieses Prozesses aufzufassen.

\section{Stadium 6.}

(Hierzu Figur 6-8.)

Embryo von Hypogeophis rostratus, dessen Oberflăchenbild der Abbildung 38 von $\mathrm{Br}$ auer entspricht. Horizontalschnittserie zu $8 \mu$.

Der linke Geruchssack wurde ebenfalls bei 150facher Vergrösserung modelliert.

Der Vergleich des Oberflächenbildes dieses Modelles mit dem beim vorigen Stadium beschriebenen ergiebt einen eingreifenden Unterschied. Der laterale Stirnfortsatz ist mit dem medialen vollstăndig verschmolzen, die Grenze $z$ wischen beiden ist an der Aussenfläche des Modells kaum mehr angedeutet. Nur am Gaumendach ist das beim vorigen Stadium beschriebene Grübchen noch zu erkennen.

Die Geruchsplatte hat, vorwiegend im Querdurchmesser, erheblich an Umfang zugenommen. Die bisher zum grossen Teil offene Grube ist durch Verwachsen ihrer Ränder in einen Blindsack mit elliptischem Lumen verwandelt, dasselbe mündet am oralen Pol durch eine relativ enge Oeffnung, die Apertura externa, nach aussen.

Die mediale Wand des so entstandenen Geruchsackes wird in ganzer :Ausdehnung von vielschichtigem Sinnesepithel gebildet, die laterale von einer dünneren Schicht epithelialer Zellen, die z. T. allem Anschein nach Deckepithelien entsprechen, die beim Schluss der Rander in die Tiefe verlagert wurden.

Bei Betrachtung der Innenseite des Modells sieht man vom unteren Pol des Geruchssackes einen Epithelstrang (Fig. 7 St) mundwärts ziehen und in das Epithel des Gaumendaches übergehen, gerade an der Stelle, die dem an der Unterfläche des Modells sichtbaren Grübchen entspricht.

Dieser Strang ist, wie die weitere Betrachtung des Modells und die Durchsicht der Schnittserie lehrt, zum grössten Teil solid, nur die mit der Geruchsplatte unmittelbar zusammenhăngende Partie zeigt ein feines Lumen, das mit dem der Nasenhöhle kommuniziert, und zwar mit dem am meisten dorsal ge- 
legenen Teil derselben (Fig. 8 1). Der Strang besteht sowohl in dem soliden wie auch in dem kanalisierten Teil aus einer doppelten Schicht konzentrisch angeordneter Zellen von kubischer Form. Dieselben sind auch da, wo sie mit der Geruchsplatte zusammenhängen, scharf von den Zellen der letzteren zu unterscheiden, sind also keine Sinneszellen.

Dieser Fpithelstrang stellt einen Rest der beim vorigen Stadium beschriebenen Epithelleiste (Fig. 4 F) dar, und zwar entspricht er dem am meisten medial gelegenen Teil derselben. Der lateral von dem Pfeil gelegene Teil (Fig. 4) ist zu Grunde gegangen, zwischen Geruchsack und Gaumendach erscheint deshalb am Modell (Fig. 7) eine breite Lücke, die in Wirklichkeit durch Bindegewebe ausgefüllt ist.

Der beschriebene Strang bildet, wie aus dem Gesagten hervorgeht, eine Verbindung zwischen dem Epithel des Gaumendachs und dem der Geruchsplatte, das Lumen des Geruchssackes kommuniziert jedoch einstweilen noch $\mathrm{nicht}$ mit dem der Mundhöhle.

\section{Stadium 7.}

Embryo von Ichtbyophis glutinosus, etwa gleich Fig. 38 Taf. 4 des Sarasin'schen Werkes. Horizontalschnittserie.

Die Nasenhöhle befindet sich in einem etwas weiter vorgeschrittenen Entwicklungsstadium, als bei dem zuletzt beschriebenen Hypogeophis-Embryo.

Der Geruchssack selbst zeigt lieine wesentlichen Unterschiede gegenüber dem Stadium 6, die Apertura externa ist anscheinend noch etwas enger als bei diesem. Dagegen ist bezüglich des Stranges, ler vom oralen Pol der Geruchsplatte zum Mundhöhlendach zieht, eine wichtige Aenderung zu verzeichnen. Wăhrend derselbe beim Stadium 6 in seinem grössten Teil solid war, ist er jetzt in ganzer Ausdehnung von einem Lumen durchzogen, das einerseits mit dem Hohlraume des Geruchssackes, andererseits mit dem der Mundhöhle kommuniziert. Dadurch ist also eine Verbindung zwischen beiden hergestellt, die p rimi tive Choane. Der neu entstandene Kanal entspricht dem Nasenrachengang Brauers.

Auch noch in einem zweiten Punkt ist ein bemerkenswerter Unterschied wahrnehmbar. Während der beim vorigen Stadium beschriebene Strang ganz aus zweischichtigem, kubischem Epithel 
bestand, ist bei dem vorliegenden Embryo nur in der der Mundhöhle zunächst liegenden Partie das Lumen von solchem Epithel eingeschlossen, während es im übrigen von mehrschichtigem: Epithel begrenzt wird, das mit dem der Geruchsplatte grosse Aehnlichkeit hat. Es stammt wohl auch von diesem ab und ist dadurch in den Nasenrachengang verlagert, dass beim Wachstum des Embryo der obere Teil des Geruchssackes in die Länge gezogen wurde.

2. Zusammenfassung der Ergebnisse und Vergleich mit Angabe in der Literatur.

Im Stadium 3 stellt die Nasenanlage eine flache Grube dar, die am dorsalen Pol einen kleinen Blindsack bildet, wăhrend sie oralwärts flach ausläuft. Die beiden Stirnfortsătze, die zunächst vollständig getrennt sind, schieben sich einander entgegen und verschmelzen allmälig. Die Grenze zwischen beiden ist zunăchst noch durch eine aus zwei Epithellagen bestehende Faltemarkiert, die wahrscheinlich dem Sinnesepithel der beiden Fortsătze cf. p. 375) entspricht, während das Teloderm zu Grunde gegangen ist. Ein Spalt oder ein Lumen zwischen den beiden Epithellamellen ist nicht vorhanden. Die Falte verbindet den unteren Pol der Geruchsplatte mit dem Gaumendach. Indem die Verschmelzung der Stirnfortsătze weiter schreitet, entsteht aus der Geruchsgrube durch Verwachsen der Rănder ein Geruchssack mit enger Apertura externa, die am unteren Pol liegt. Die Epithelieiste zwischen Geruchsplatte und Gaumendach verschwindet bis auf ibren medialen Teil, der als solider Strang bestehen bleibt. Etwas später erhalt derselbe ein Lumen, das einerseits mit dem des Geruchssackes kommuniziert, andererseits in die Mundhöhle mündet, also eine primitive Choane darstellt. Ich habe mit $\mathrm{Brauer}$ diesen Kanal als $\mathrm{Nasen-}$ rachengang bezeichnet.

Meine mikroskopischen Befunde, die durch zwei Modelle plastisch dargestellt wurden, decken sich in der Hauptsache mit den oben angeführten Schlüssen, die Brauer aus der Betrachtung des Oberflächenbildes zog. Nur in einem Punkte divergieren unsere Resultate: $\mathrm{Brauer}$ nimmt an, dass, wăhrend medialer und lateraler Stirnfortsatz sich entgegen wachsen, zwischen beiden die Nasenrachenrinne als enger Spalt. bestehen bleibt, und, nachdem erstere vollständig verschmolzen, 
einen Verbindungskanal zwischen Mund und Nasenhöhle darstellt. Ich konnte auf Grund des mikroskopischen Befundes fesstellen, dass ein solcher Kanal anfangs ni cht vorhanden ist, sich vielmehr erst später in einem Zellstrang bildet, der zunächst. solid ist.

Die Natur macht also hier, indem sie eine zuerst vorhandene Verbindung verschliesst, dann wieder öffnet, einen Umweg, der sich bei Betrachtung des Oberflächenbildes allerdings nicht vermuten lässt, an dessen Vorhandensein jedoch auf Grund des mikroskopischen Befundes nicht gezweifelt werden kann.

3. Vergleich $z$ wischen Gymnophionen und den übrigen Amphibien.

Die Bildung des Nasenlumens und der Choanen erfolgt bei den Gymnophionen in vollstandig anderer Weise, als bei den in Teil eins und zwei beschriebenen Amphibiengattungen.

Die Verwachsung der Ränder der Geruchsgrube spielt bei ihnen bezügl. der Lumenbildung eine bedeutend grössere Rolle als bei den Anuren und Urodelen, bei denen 'der Hauptteil des Lumens durch Dehiscenz der Zellen der Geruchsplatte entsteht.

Viel tiefgreifender ist jedoch noch der Unterschied in Bezug auf die Choanenbildung. Während bei Anuren und Urodelen eine Verbindung zwischen Mundbucht und der ersten Anlage der Geruchsgrube vollkommen fehlt, und die Choanen dadurch ententstehen, dass zunächst der Geruchssack in der im ersten Teil ausführlich beschriebenen Weise mit dem Mundhöhlenepithel verwåchst, dann das Nasenlumen in die Mundhöhle durchbricht, bildet sich bei den Gymnophiouen schon sehr frühzeitig eine Kommunikation zwischen Mundbucht und Nasenhöhle, indem letztere oralwärts flach ausläuft. Diese Verbindung wird zunächst wenigstens äusserlich durch Verwachsung der beiden Stirnfortsätze aufgehoben, erst auf Umwegen entsteht wie oben beschrieben, die primitive Choane in Form des Nasenrachenganges. Der Entstehungsmechanismus derselben ist also von dem Bildungsmodus der Choanen bei den übrigen Amphibien. sehr verschieden.

Ebenso verschieden ist das Resultat. Während bei Anuren und Urodelen das Sinnesepithel unmittelbar mit dem indifferenten Epithel des entodermalen Vorderdarms in Verbindung tritt, ist. bei den Blindwühlen ein vom Ektoderm abstammender Kanal, 
der zum ektodermalen Gaumendach führt, der Nasenrachengang, eingeschaltet.

Wodurch diese tiefgreifenden Unterschiede in der Choanenbildung bei den verschiedenen Gliedern der Amphibiengruppe bedingt sind, lässt sich wohl nur ganz allgemein vermuten; sie sind wohl als Anpassung an die so grundverschiedenen Lebensbedingungen anzusehen, die ja auch im Uebrigen beim Aufbau der Organe măchtig modifizierend eingewirkt haben. Wie dieselben im speziellen die Entwicklung des Geruchsorgans beeinflusst haben, entzieht sich einstweilen noch unserer Beurteilung.

Bei oberflachlicher Betrachtung könnte es scheinen, als habe die Choanenbildung der Gymnophionen mehr Aehnlichkeit mit der, wie wir sie bei den Mammaliern sehen, als mit der bei den übrigen Amphibien. Auch bei jenen spielt das Verwachsen der beiden Stirnfortsätze eine gewisse Rolle, dieselbe ist jedoch, wie man bei genauerem Zusehen leicht feststellt, bei beiden Gattungen sehr verschieden, da bei den Säugern die beiden Falten die Sinnesgrube $z$ w ischen sich fassen, wăhrend bei den Gymnophionen die Geruchsgrube ganz im Bereich des medialen Stirnfortsatzes liegt.

\section{Weitere Entwicklung der Nasenhöhle und Entstehung der Blindsackbildungen.}

\section{a. Eigene Beobachtungen als Ergänzung der Sarasin'schen Befunde. Stadium 8.}

Embryo von Ichthyophis glutioosus, ungefähr Fig. 43, Taf. V des S a rasin 'schen Werkes entsprechend.

Der Kopf hat eine spitze Form angenommen. Die Apertura externa, die im letzten Stadium noch ziemlich weit von der Kopfspitze entfernt an der ventralen Seite lag, ist ganz an die Spitze gerückt, an der sie ebenfalls ventral liegt. Dadurch ist ein langer, aus zweischichtigem, cubischem Epithel bestehender Einführungsgang entstanden. Derselbe mündet von ventral und lateral her in den apicalen Pol des eigentlichen Geruchssackes. Dieser stellt einen länglichen Hohlraum dar, dessen Querschnitt in den vorderen Partien rundlich, in den caudalen elliptisch ist. Seine Wand wird zum grössten Teil von einer måchtigen Platte 
von Sinnesepithel gebildet, nur ein kleines Stückchen derselben besteht aus zweischichtigem, indifferentem Epithel. Dieses. liegt fast in ganzer Ausdehnung des Geruchssackes lateral, nur am caudalen Ende desselben ventro-lateral. Der eigentliche. Geruchssack trägt an seiner ventro-lateralen Seite eine aus. Sinnesepithel bestehende Ausstülpung, die in Form und Lagevollkommen dem Jakobson'schen Organ der Sarasin (cf. Fig. 29, 30, 32, 33 Heft 4, Tafel 17) entspricht. Auch bei Durchsicht der vorliegenden Serie (von der Kopfspitze beginnend). lässt sich dentlich verfolgen, wie die Ausstülpung, die, wie ge-sagt, in ihrem grössten Theil ventro-1ateral vom Geruchssack liegt, ziemlich plötzlich auf die ventro-mediale Seite. herüberrückt, sodass ihr caudaler Pol medial vom Nasenrachen-gang liegt. - Ich möchte für diesen Blindsack jedoch nicht die von den Sarasin gewählte Bezeichnung "Jakobson'sches. Organ" anwenden, vielmehr möchte ich ihn zunächst, wie bei der Beschreibung der ähnlichen Bildungen bei Anuren und Urodelen, „unteren Blindsack “ nennen. Am vorderen Pol desselben liegt ein kleines Drüsenconglomerat, die „untere Nasendrüse („Jakobson'sche Drüse“ der Sarasin).

Mit der Mundhöhle kommuniziert die eigentliche Nasenhöhle durch einen ziemlich langen, aus zweischichtigem Epithel bestehenden Gang. Derselbe stellt offenbar eine weitere Entwicklungsstufe des beim vorigen Stadium beschriebenen Nasenrachenganges dar. Er mündet in den caudalen Pol der Nasenhöhle von ventro-lateral her, und zwar in die aus indifferentem Epithel bestehende Wandpartie. Am Gaumendach endigt der Gang in Form eines schmalen Spaltes, der sich noch eine Strecke weit caudalwärts als Furche verfolgen lässt.

Das zuletzt beschriebene Stadium stimmt ziemlich genau mit dem jüngsten der von den Sarasin bezüglich der Nasenhöhle untersuchten Exemplare überein. Die späteren Entwicklungsphasen bis zum erwachsenen Zustand sind von den genannten Forschern ziemlich lückenlos dargestellt, sodass weitere Untersuchungen in dieser Richtung kaum nötig sind. Ich habe bei Durchsicht der mir von Herrn Dr. Peter zur Verfügung gestellten Serien dieselben Resultate erhalten. Wenn ich demnach auch nichts wesentlich neues bringen kann, möchte ich doch der Vollständigkeit wegen in kurzen Zügen die weitere Entwicklung 
der Nasenhöhle skizzieren, unter Hinweis auf die ausführlichen Darstellungen in dem ausgezeichneten Sarasin's chen Werke (Heft 4, p. 176-193 und Tafel 16 u. 17).

Die Hauptnasenhöhle nimmt im Lauf der weiteren Entwicklung, besonders in den caudalen Partien, an Breite bedeutend $\mathrm{zu}$, dabei wird sie von der ventralen Seite her eingebuchtet, sodass sie auf der Unterseite eine tiefe Längsfurche erhält, die einem unter dem Boden der Nasenhöhle liegenden Knocbenwulst entspricht. Gleichzeitig tritt eine Sonderung der Höhle in einen medialen, Sinnesepithel tragenden Abschnitt ein, und in einen lateralen, der von indifferentem Epithel begrenzt ist. Der "untere Blindsack", bezw. das „Jakobson'sche Organ" bildet sich zu einem langen Schlauch aus, der beim erwachsenen Tier dicht vor der Choane von der Haupthöhle sich abzweigt, ein kurzes Stückchen parallel derselben verläuft, dann rechtwinklig umbiegt und in seinem Hauptteil quer zur Längsachse der eigentlichen Nasenhöhle gestellt ist. - Ganz gegen Ende der Larvenperiode tritt der Thrănennasengang mit dem blinden Ende des „Jakobson'schen Organs" in Verbindung.

Ich möchte diese kurz referierten Befunde der Sarasin nur in Bezug auf den Einführungsgang und den Nasenrachengang durch einige eigene Beobachtungen ergănzen.

Der letztere nimmt gegen Ende des Embryonallebens schnell an Ausdehnung ab, bei einer Larve von $8,8 \mathrm{~cm}$ ist er kaum mehr als solcher vorhanden, vielmehr mündet er vermittelst einer kurzen, weiten Choane in die Mundhöhle (vergl. auch Sarasin, Tafel 17, Figur 33 und Tafel 16, Figur 18).

Der Choanalschleimbeutel der Sarasin ist schon sehr frühzeitig in Gestalt einer kleinen, lateralwärts gerichteten Ausbuchtung angedeutet, die vom Nasenrachengang kurz vor seiner Mündung in die Nasenhöhle abgeht.

Die Apertura externa, und mit ihr der Einführungsgang, wandert allmälig von der ventralen Seite der Kopfspitze an die laterale, dann an die dorso-laterale Seite, dabei wird der Einführungsgang immer kürzer, bei einer Larve von $16 \mathrm{~cm}$ Länge ist er kaum mehr vorhanden, vielmehr gelangt man durch die Apertura externa unmittelbar in die Hauptnasenhöhle (cf. Sarasin: Tafel 17, Figur 27). 


\section{b. Vergleich der Befunde bei den verschiedenen Amphibiengattungen.}

Wie bei der Gegenüberstellung von Urodelen und Anuren, so nimmt auch beim Vergleich der bei den Gymnophionen erhobenen Befunde mit denen bei den übrigen Amphibien vorwiegend ein Gebilde unser Interesse in Anspruch, das ich bei allen als "unteren Blindsack" bezeichnet habe. P. und F. Sarasin, Seydel, Burckhardt u. a. haben bereits darauf hingewiesen, dass die Anlage desselben bei den Blindwühlen in der gleichen Weise erfolge, wie bei den Urodelen, und haben daraus den Schluss gezogen, dass die entsprechenden Organe bei den verschiedenen Amphibiengattungen homolog zu setzen seien.

Ich habe die Befunde dieser Autoren vollkommen bestătigen können, und balte auf Grund des Befundes bei der ersten Anlage den unteren Blindsack der Gymnophionen ebenfalls für homolog dem der Anuren und Urodelen, wenn auch die Entstehung des Nasenlumens und das fertig ausgebildete Geruchsorgan bei den verschiedenen Gattungen weitgehende Unterschiede aufweist.

Bildungen, die den übrigen Blindsäcken der Schwanzlurche und Anuren entsprächen, fehlen bei den Blindwühlen, andererseits vermissen wir bei jenen ein Homologon für den Choanenschleimbeutel der Gymnophionen.

\section{Homologie zwischen unterem Blindsack der Amphibien und Jacobson'schem Organ der Amnioten.}

Ich habe bereits mehrfach erwăhnt, dass von einer Reihe von Autoren wie Fleischer, Seydel, P. u. F. Sarasin und Burckhardt der "untere Blindsack ${ }^{\star}$ der Amphibien als Jakobson'sches Organ aufgefasst wird, und zwar auf Grund verschiedener Thatsachen. Erstens erfolgt die erste Anlage des unteren Blindsacks in ahnlicher Weise, wie die des Jacobson'schen Organs der Amnioten, nămlich durch eine zunächst medial gerichtete Ausstülpung des Sinnesepithels der Nasenhöhle. Ferner stehen beide mit einer Drüse in Verbindung, die früher angelegt wird als die übrigen Drüsenbildungen der Nasenhöhle. Drittens endlich werden beide vom ventralen Ast des Olfactorius innerviert. 
Ich muss zugeben, dass diese Thatsachen, die auch ich bei meinen Untersuchungen vollkommen bestätigen konnte, den Gedanken an eine Homologie sehr nahe legen.

Ich glaube jedoch, dass man bei der Beurteilung dieser Frage einen überaus. wichtigen Punkt nicht unberücksichtigt. lassen darf: den grossen prinzipiellen Unterschied in der Anlage und Entwicklung der Nasenhöhle bei Amphibien einer- und Amnioten andererseits. Die Bildung des Nasenlumens und derChoanen ist bei den genannten Gruppen so grundverschieden, dass wir sie wohl kaum als Modifikation eines gemeinsamen Grundtypus ansehen können. Sie stellen vielmehr zwei Entwicklungstypen dar, die kaum eine Aehnlichkeit aufweisen.

Hat sich nun aber die Nasenhöhle der Amphibien in einer Weise entwickelt, die von der bei den Amnioten beobachteter so ganz und gar abweicht, dann sind wir wohl kaum berechtigt, an ihnen auftretende Gebilde als homolog aufzufassen, selbst wenn sie mancherlei Aehnlichkeiten aufweisen. Wir haben vielmehr die Möglichkeit zu berücksichtigen, dass sich bei beiden, auch ohne dass eine phylogenetische Grundlage vorhanden ist, ähnliche Gebilde entwickelt haben, vielleicht sogar einer ähnlichen Funktion dienend. Derartige Organe müssen wir dann aber als analoge, nicht als homologe, bezeichnen.

Ferner ist meines Erachtens zu berücksichtigen, dass der untere Blindsack der Amphibien sich ontogenetisch wesentlich später entwickelt, als das Jacobson'sche Organ der Amnioten, das bekanntlich sehr früh, schon vor dem Schluss der Nasenrinne, angelegt ist.

Unter diesen Umstanden ist es bei dem heutigen Stand unserer Kenntnisse vielleicht vorsichtiger, für Jakobson'sches Organ der Amnioten und unteren Blindsack der Amphibien eine Ana$\log$ i e, nicht $\mathrm{Homolog}$ i e, anzunehmen, bis etwa weitere Grundlagen für die Beurteilung der Frage gewonnen sein werden.

\section{Verzeichnis der benutzten Literatur.}

Ausser der in Teil 1 und 2 (dieses Archiv Bd. 58, p. 478) angeführten Literatur wurde benutzt:

22. Peter, K. : Zur Bildung des primitiven Grumens bei Mensch u. Säugetieren. Anatom. Anzeige: 1902. 


\section{Figurenverzeichnis Tafel XIX.}

Fig. 1. Schnitt durch die Mitte der linken Geruchsplatte eines Embryo von Hypogeophis rostratus, Stadium I. Vergrösserung c. 150fach. Bei $x$ zu Grunde gehende Zellen der Deckschicht.

Fig. 2. Horizontalschnitt durch die linke Geruchsplatte eines Embryo von Hypogeophis rostratus, Stadium 3. Vergrösserung 80fach.

Fig. 3 u. 4. Modell des linken Geruchssackes und seiner Umgebung von einem Embryo von Hypogeophis rostratus, in Fig. 3 von unten und aussen, in Fig. 4 von innen gesehen.

Fig. 5. Horizontalschnitt durch den unteren Pol der Geruchsplatte desselben Embryo, Vergrösserung 80fach.

Fig. 6 n. 7. Modell des linken Geruchssackes von einem Embryo von Hypogeophis rostratus, Stadium 6, in Fig. 6 von aussen und unten, in Fig. 7 von innen gesehen.

Fig. 8. Horizontalschnitt durch den unteren Pol eines Embryo von Hypogeophis rostratus, Stadium 6, Vergrösserung 80fach. Das HauptIumen der Nasenböble ist in dem gezeichneten Schnitt nicht getroffen, sodass der Zusammenhang desselben mit dem Lumen (1.) des Epithelstranges (St.) nicht ersichtlich ist.

Leider sind einige Figuren in falscher Stellung lithographiert worden; es geben die angebrachten Bezeichnungen Auskunft über die Lage derselben.

\section{Buchstabenerklärang.}

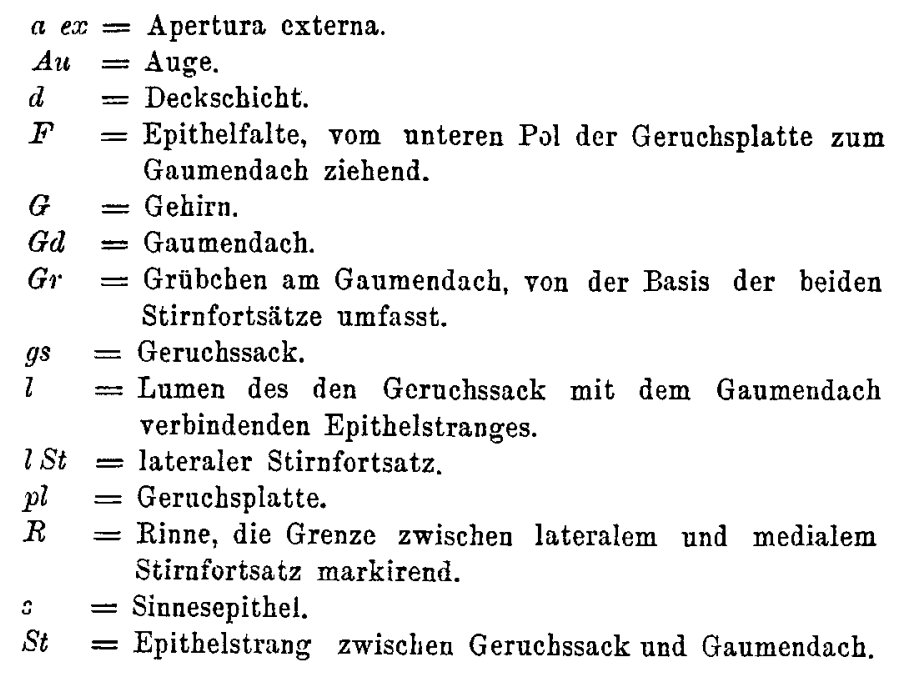

